\title{
Chronic Kidney Disease in Primary Care
}

\author{
Duaine D. Murphree, MD, and Sarah M. Thelen, MD
}

Because chronic kidney disease is a growing health concern, family physicians must be equipped to care for this unique patient population. Diabetes mellitus and hypertension, which are commonly addressed in the office setting, are the largest contributors to chronic kidney disease; therefore, these risk factors should be tightly controlled and these patients should be screened closely for signs of renal damage. The National Kidney Foundation recommends that screening include determination of the glomerular filtration rate (GFR) and assessment for the presence of proteinuria. Once the diagnosis of chronic kidney disease is established (by the presence of persistent kidney damage or a GFR $<60 \mathrm{~mL} / \mathrm{min} / 1.73 \mathrm{~m}^{2}$ for at least 3 months), the etiology of chronic kidney disease needs to be elucidated. Often the etiology can be determined by history alone; however, reversible causes of chronic kidney disease should be considered in all patients. Regardless of the underlying etiology of the chronic kidney disease, the family physician can make a significant impact in slowing the progression of chronic kidney disease through strict blood pressure control, tight glycemic control, reduction in the degree of proteinuria, and smoking cessation. All chronic kidney disease patients are at significantly increased risk of cardiovascular events; therefore, additional cardiovascular risk factors such as hyperlipidemia should be managed aggressively. Assessment for the complications of chronic kidney disease, including anemia, bone metabolism abnormalities, metabolic acidosis, and malnourishment, should be assessed once the GFR declines below $60 \mathrm{~mL} / \mathrm{min} / 1.73 \mathrm{~m}^{2}$ (stage 3). Early screening and treatment of these complications can prevent the development of further sequelae and should not be delayed until referral to nephrology. Appropriate counseling and health maintenance is also needed for this patient population and should be given by the family physician involved in the patient's care. (J Am Board Fam Med 2010;23: 542-550.)

Keywords: Chronic Kidney Disease, Kidney Disorders, Glomerular Filtration Rate, Clinical Review

The prevention, early detection, and prompt treatment of chronic kidney disease is within the realm of the family physician. It is estimated that $13 \%$ of the adult population suffers from chronic kidney disease and the numbers are expected to continue to climb. ${ }^{1}$ With this rise in the prevalence of chronic kidney disease, the role of the family physician in improving patient care and disease out-

This article was externally peer reviewed.

Submitted 28 May 2009; revised 1 March 2010; accepted 8 March 2010.

From St. Vincent's Family Medicine Residency, Jacksonville, FL (DDM); and Family Medicine Residency, University of Alabama in Huntsville (SMT).

Funding: none.

Conflict of interest: none declared.

Corresponding author: Duaine D. Murphree, MD, St. Vincent's Family Medicine Residency, Faculty, 2627 Riverside Avenue, Jacksonville, FL 32204 (E-mail: dmurp002@ stvincentshealth.com). comes has become increasingly evident. In this regard, additional training and education about chronic kidney disease and its complications is warranted to better equip family physicians to directly impact disease progression. It has been shown that primary care physicians' familiarity with chronic kidney disease is suboptimal, ${ }^{2}$ and the goal of this article is to provide family physicians with the knowledge required to provide quality care for their patients with chronic kidney disease.

Chronic kidney disease is defined by the $\mathrm{Na}$ tional Kidney Foundation ${ }^{3}$ as either a decline in glomerular filtration rate (GFR) to $<60 \mathrm{~mL} / \mathrm{min} /$ $1.73 \mathrm{~m}^{2}$ or the presence of kidney damage for at least 3 months. Signs of kidney damage classically include proteinuria but other markers of damage, such as persistent glomerulonephritis or structural damage from polycystic kidney disease, can also be present. Chronic kidney disease has been subdi- 
Table 1. Classifications of Chronic Kidney Disease

\begin{tabular}{lc}
\hline Stage & Associated GFR $\left(\mathrm{mL} /\right.$ minute $\left./ 1.73 \mathrm{~m}^{2}\right)$ \\
\hline 1 & $>90$ with persistent kidney damage* \\
2 & $60-89$ with persistent kidney damage* \\
3 & $30-59$ \\
4 & $15-29$ \\
5 & $<15$ \\
\hline
\end{tabular}

*Kidney damage includes both functional damage (proteinuria, glomerulonephritis) and structural damage (polycystic kidneys). GFR, glomerular filtration rate.

vided into 5 stages of increasing severity (Table 1). The complications of chronic kidney disease are associated with various stages and the screening recommendations vary by stage. Previous laboratory evaluations of renal function, such as 24-hour urine collections for creatinine clearance, are generally no longer necessary because of the accuracy and ease of the calculation of the GFR from serum laboratory values. The use of serum creatinine is not sufficient for determining chronic kidney disease because approximately half of the renal function must be lost before the creatinine will be elevated out of the normal range.

GFR calculations should be included routinely by laboratories; however, if needed, it can be easily calculated using the Modification of Diet in Renal Disease study calculator. This is the equation that is recommended by that National Kidney Foundation $^{3}$ and is available online. ${ }^{4}$ The Cockroft-Gault equation is useful for medications that require renal dosing because it is used to calculate creatinine clearance. Neither of these calculations should be used during acute renal failure because a stable creatinine level is required to ensure their accuracy.

\section{Prevention}

Knowledge of the risk factors for the development of chronic kidney disease is crucial to prevention of the disease process. Diabetes mellitus and hypertension are the leading causes of chronic kidney disease; therefore, these risk factors must be tightly controlled in all patients. Glycemic and hypertensive control is discussed in more detail later in this review. In addition, medication lists should be reviewed closely with patients to ensure that any potentially nephrotoxic medications are being used appropriately and in correct dosages. In particular, over-the-counter medications should be evaluated for the potential of drug interactions and nephrotoxicity. Nonsteroidal anti-inflammatory drugs (NSAIDs) are notoriously nephrotoxic and physicians may underestimate their patient's usage of these medications unless they specifically inquire.

\section{Screening: Whom and How?}

The patients who are at increased risk of chronic kidney disease are those with long-standing diabetes and/or hypertension; these conditions are the primary contributors to kidney disease. The $\mathrm{Na}$ tional Kidney Foundation ${ }^{3}$ has identified the following additional risk factors for chronic kidney disease: $>60$ years old, racial or ethnic minorities, exposure to known nephrotoxins, low income or education level, autoimmune diseases, systemic infections, urinary tract infections, nephrolithiasis, neoplasia, family history of kidney disease, recovery from acute renal failure, reduction in kidney mass, and low birth weight.

The US Preventive Services Task Force currently has no specific screening recommendations for kidney disease. ${ }^{5}$ The National Kidney Foundation, however, recommends testing for all patients with diabetes, hypertension, a family history of kidney disease, age $>60$ years, and ethnic minorities because these are the most prominent risk factors for chronic kidney disease. ${ }^{3}$

For these patients who are at increased risk for chronic kidney disease, it is recommended that the minimal screening for kidney damage include assessment of GFR and proteinuria. Currently, the guidelines for hypertension from the Seventh Report of the Joint National Committee on Prevention, Detection, Evaluation, and Treatment of High Blood Pressure guidelines ${ }^{6}$ recommend an annual screening urinalysis to assess proteinuria; however, the National Kidney Foundation ${ }^{3}$ and the European Society of Cardiology/European Society of Hypertension ${ }^{7}$ recommend screening for microalbuminuria. The European Society of Cardiology/European Society of Hypertension state in their 2007 Guidelines for the Management of Arterial Hypertension: "Microalbuminuria has now been considered an essential component in the assessment of organ damage because its detection is easy and relatively inexpensive." ${ }^{77}$ A routine urinalysis detects only protein unless an albumin-specific dipstick is used. When the glomerular membrane is damaged, the initial protein that is spilled into the 
urine is albumin because of its molecular size and negative charge. Therefore, screening for the presence of microalbuminuria is the more sensitive test for detection of early kidney damage. The American Diabetic Association ${ }^{8}$ recommends that all diabetic patients have an annual screen for microalbuminuria. Microalbuminuria is considered positive when the level is $>30 \mathrm{mg} / \mathrm{g}$; however, there are gender-specific values that have not entered into routine use at this point $(>17 \mathrm{mg} / \mathrm{g}$ in men and $>25 \mathrm{mg} / \mathrm{g}$ in women). If the level of proteinuria exceeds $500 \mathrm{mg} / \mathrm{g}$, using an untimed (spot) urine protein/creatinine ratio is recommended to assess the severity of the proteinuria and its response to interventions. Currently, there is agreement among the recommendations for assessment of renal function with screening chemistry and calculated GFR., ${ }^{3,6-8}$

\section{Etiology}

Chronic kidney disease can be a manifestation of another chronic illnesses that are causing end-organ renal damage, such as diabetes mellitus or hypertension. Alternatively, chronic kidney disease can be an intrinsically renal disease, such as polycystic kidney disease. Therefore, at diagnosis of chronic kidney disease, family physicians must determine the underlying etiology so that the treatment plan can be appropriately directed.

Diabetes is the most prominent cause of chronic kidney disease, accounting for $33 \%$ of adult chronic kidney disease cases. ${ }^{3}$ Conversely, 20\% to $40 \%$ of diabetics will develop diabetic nephropathy during the course of their disease ${ }^{8}$; therefore, as the number of diabetic patients increases, the incidence of chronic kidney disease can be expected to follow. Diabetic nephropathy follows a classical progression that, when present, often negates the need for a renal biopsy to confirm its presence. The initial presentation of diabetic kidney disease is microalbuminuria followed by increasing severity of proteinuria as the glomerular filtration membrane is further damaged. The development of hypertension subsequently occurs, followed by a decline of the GFR. ${ }^{3}$ Both type 1 and type 2 diabetes can cause kidney disease; however, because type 2 diabetes is increasingly prevalent, it is more commonly associated with kidney damage than type 1 . The onset of diabetic kidney disease closely correlates with the onset of diabetic retinopathy because both are manifestations of microvascular disease ${ }^{9}$; therefore, if retinopathy is not present in a diabetic patient with chronic kidney disease, the nephropathy may not be caused by the diabetes, and other etiologies should be thoroughly evaluated. ${ }^{8}$

Vascular disease (primarily hypertension) is the second most common cause of chronic kidney disease (it causes $21 \%$ of adult chronic kidney disease cases). ${ }^{3}$ Hypertensive nephrosclerosis is associated with addition signs of hypertensive end-organ damage because of long periods of poorly controlled hypertension. Atherosclerotic renovascular disease is suggested by a sudden worsening of hypertension, with findings of atherosclerosis in non-renal areas. Renal ultrasound may show asymmetrical kidney sizes, with the smaller kidney receiving less blood supply because of its renovascular disease. There are multiple imaging studies that can be used to confirm suspected renal artery stenosis. Duplex Doppler ultrasonography is useful as an initial screening test when renal artery stenosis is suspected in a patient with chronic kidney disease because it does not require administration of intravenous dye. Magnetic resonance angiography with gadolinium must be used judiciously in patients with a GFR $<60 \mathrm{~mL} \cdot \mathrm{min} / 1.73 \mathrm{~m}^{2}$ because of the risk of nephrogenic systemic fibrosis. Similarly, computed tomographic angiography may worsen renal function and should be used cautiously. Renal angiography is the gold standard for confirmation and is useful for therapeutic interventions; however, it also carries the risk of worsening renal function. ${ }^{10}$ Among young women with no findings of atherosclerosis, fibromuscular dysplasia should be considered as the etiology of renal artery stenosis.

Differentiation between the various etiologies of kidney damage can be difficult at times and, in all cases, the presence of treatable causes should be assessed. This evaluation begins with a detailed history and physical, with consideration of the broad differential of causes of chronic kidney disease as illustrated in Table 2. If the etiology cannot be elucidated, then consultation with a nephrologist should be considered because a renal biopsy may be indicated.

\section{Interventions to Slow the Progression of Kidney Disease}

The Modification of Diet in Renal Disease study ${ }^{11}$ followed chronic kidney disease patients at all 


\begin{tabular}{|c|c|}
\hline Diagnosis & Clinical Indicators \\
\hline Diabetes mellitus & $\begin{array}{l}\text { Classical clinical course of microalbuminuria, followed by clinical proteinuria, hypertension, } \\
\text { and then declining GFR. }\end{array}$ \\
\hline Hypertension & $\begin{array}{l}\text { Usually characterized by severely elevated blood pressure readings over a long period, with } \\
\text { associated end-organ damage in addition to kidney disease. }\end{array}$ \\
\hline Nephrotoxic medications & $\begin{array}{l}\text { Review prescribed and over-the-counter medications as well as intravenous contrast dye or } \\
\text { gadolinium exposure. }\end{array}$ \\
\hline Systemic lupus erythematosus & $\begin{array}{l}\text { Evaluate for photosensitivity, malar/discoid rashes, oral ulcers, arthritis, serositis, neurological } \\
\text { symptoms, hematological findings, ANA/dsDNA positive. }\end{array}$ \\
\hline HIV nephropathy & Signs and symptoms of immunodeficiency; HIV positive on testing. \\
\hline Congestive heart failure & $\begin{array}{l}\text { Signs and symptoms of heart failure present. Because fluid overload is common in chronic } \\
\text { kidney disease, diagnosis is made through echocardiogram to evaluate systolic and diastolic } \\
\text { heart function. }\end{array}$ \\
\hline Genetic syndromes & Evaluation of family history is suggestive. \\
\hline Hepatorenal syndrome & $\begin{array}{l}\text { History or evidence of cirrhosis with resultant portal hypertension, ascites, and renal } \\
\text { vasoconstriction. Classically lack significant proteinuria. }\end{array}$ \\
\hline Nephrolithiasis & $\begin{array}{l}\text { Evaluate for history of hematuria and symptoms of renal colic. Long-standing obstruction } \\
\text { can cause permanent renal impairment. }\end{array}$ \\
\hline Benign prostatic hypertrophy & $\begin{array}{l}\text { Evaluate male patients for hesitancy, straining, or weak flow during urination and nocturia. } \\
\text { Confirm with prostate exam. }\end{array}$ \\
\hline Glomerulonephritis & $\begin{array}{l}\text { Broad category of diseases including postinfectious (streptococcal) as well as various vasculitis } \\
\text { diseases. Urinalysis suggestive with presence of red blood cell casts. }\end{array}$ \\
\hline
\end{tabular}

GFR, glomerular filtration rate; ANA, antinuclear antibodies; dsDNA, double-stranded deoxyribonucleic acid; HIV, human immunodeficiency virus.

stages for a 2-year period and concluded that $85 \%$ of patients had a decline in their GFR, with the average rate of decline $4 \mathrm{~mL} / \mathrm{min}$ annually regardless of the baseline GFR. There are modifiable and nonmodifiable factors that contribute to this decline. These factors have been shown to be significant regardless of the underlying etiology of the chronic kidney disease. In general, the nonmodifiable risk factors associated with more rapid decline in kidney disease include increased age, AfricanAmerican race, and male sex. The modifiable risk factors are the focus of treatment to halt disease progression and include higher levels of proteinuria, a lower serum albumin level, higher blood pressure, poor glycemic control, and smoking. Currently there is conflicting data regarding the role of dyslipidemia and anemia in the role of kidney disease progression. ${ }^{3}$

\section{Proteinuria}

Because proteinuria contributes to an increase in renal damage, screening and quantification of the presence of proteinuria is critical in the care of chronic kidney disease patients. Random (spot) samples of urine for calculation of the urine protein-creatinine ratio eliminate the need for 24-hour urine collections for quantification of proteinuria.
Once proteinuria is identified, its control becomes a high priority. The goal of treatment is to decrease the degree of proteinuria; even low levels of proteinuria are associated with progression of chronic kidney disease and cardiovascular disease. ${ }^{3}$

Angiotensin converting enzyme (ACE) inhibitors are considered first-line medications for proteinuria, regardless of the underlying cause or stage of chronic kidney disease. ${ }^{3}$ As such, family physicians should become competent with the initiation of these medications and confidence with monitoring their effects. Because hyperkalemia and slight worsening of renal function can occur with the initiation of ACE inhibitors, these factors should be monitored; however, the medications should not be discontinued without due cause. Mild hyperkalemia (potassium $<5.6 \mathrm{mmol} / \mathrm{L}$ ) can often be controlled by dietary changes, cessation of NSAID use, and potassium-sparing diuretics, if applicable. In addition, potassium excretion can be enhanced by the addition of a loop diuretic. For hyperkalemia $>5.6$ $\mathrm{mmol} / \mathrm{L}$, the ACE inhibitor should be immediately discontinued and the patient should be treated appropriately. With regard to the concern of acute renal failure related to the initiation of ACE inhibitors, a modest rise in creatinine level $(<30 \%$ increase) within 1 to 2 weeks of initiation of therapy 
is considered acceptable. The patient should be monitored to ensure that additional rise does not occur because this would be cause for discontinuation of medication and further evaluation. Renal artery stenosis, hypovolemia, or uncompensated heart failure may be associated with a rise in creatinine level of $>30 \%$ and, once treated, the ACE inhibitor may be reinstated safely. ${ }^{12}$

An angiotensin receptor blocker (ARB) may be considered for patients who are unable to tolerate ACE inhibitors. In diabetic kidney disease, an ARB may be used as a first-line alternative to ACE inhibitors. ${ }^{7}$ In addition, the candesartan and lisinopril microalbuminuria study demonstrated the benefit of the use of the combination of an ACE inhibitor (lisinopril) with an ARB (candesartan) in patients with diabetic-associated microalbuminuria. ${ }^{13}$ The addition of a nondihydropyridine calcium channel blocker, such as diltiazem or verapamil, can further decrease the degree of proteinuria, as can the addition of a thiazide or loop diuretic. ${ }^{3}$ However, the blockade of the renin-angiotensin system remains the cornerstone of treatment of proteinuria.

\section{Blood Pressure Control}

Strict blood pressure control is a high priority in the care of the patient with chronic kidney disease. For the reasons mentioned above, ACE inhibitors or ARBs are commonly used as the initial medications to achieve blood pressure control; however, often a multidrug regimen is needed. Commonly, diuretics are needed for patients with chronic kidney disease because of the hypertensive effect of volume overload. ${ }^{14}$ According to the Seventh Report of the Joint National Committee on Prevention, Detection, Evaluation, and Treatment of High Blood Pressure guidelines, ${ }^{6}$ the goal blood pressure is $<130 / 80 \mathrm{~mm} \mathrm{Hg}$ in patients with chronic kidney disease; however, the National Kidney Foundation ${ }^{3}$ suggests a more stringent goal of $<125 / 75 \mathrm{~mm} \mathrm{Hg}$ for patients with chronic kidney disease and significant proteinuria. With the achievement of these blood pressure goals, further kidney damage can be avoided and the progression of the patient's chronic kidney disease can be slowed.

\section{Glycemic Control}

Regardless of the cause of chronic kidney disease, tight glycemic control should be achieved for all diabetic patients. The American Diabetic Associa-
Table 3. Dosage of Diabetic Medications in Chronic Kidney Disease

\begin{tabular}{|c|c|}
\hline Diabetic Medication & Renal Dosage* \\
\hline \multicolumn{2}{|l|}{ Biguanines } \\
\hline Glucophage (metformin) & Renal impairment: avoid use \\
\hline \multicolumn{2}{|l|}{ Sulfonureas } \\
\hline Glucotrol (glipizide) & $\begin{array}{l}\mathrm{CrCl}^{+}<50 \text { : decrease dose by } \\
\quad 50 \%\end{array}$ \\
\hline Diabeta (glyburide) & $\mathrm{CrCl}^{+}<50:$ avoid use \\
\hline Amaryl (glimepiride) & $\begin{array}{l}\text { Renal impairment: start } 1 \mathrm{mg} \\
\text { daily, increase slowly, } \\
\text { monitor glucose }\end{array}$ \\
\hline \multicolumn{2}{|l|}{ Glitazones } \\
\hline Actos (pioglitazone) & No adjustment \\
\hline Avandia (rosiglitazone) & No adjustment \\
\hline \multicolumn{2}{|l|}{ Alpha-glucosidase inhibitors } \\
\hline Precose (acarbose) & Creatinine $>2$ : avoid use \\
\hline Glyset (miglitol) & Creatinine $>2$ : avoid use \\
\hline \multicolumn{2}{|l|}{ Meglitinides } \\
\hline Starlix (nateglinide) & No adjustment \\
\hline Prandin (repaglinide) & $\begin{array}{l}\mathrm{CrCl} 20-40: \text { start } 0.5 \mathrm{mg} \\
\text { before every meal, use } \\
\text { titrate with caution } \\
\mathrm{CrCl}<20 \text { : not defined }\end{array}$ \\
\hline \multicolumn{2}{|l|}{ Incretin mimetics } \\
\hline Byetta (exenatide) & $\begin{array}{l}\mathrm{CrCl} \text { 30-80: no adjustment } \\
\mathrm{CrCl}<30 \text { \& } \mathrm{HD} \text { : not } \\
\text { recommended }\end{array}$ \\
\hline Januvia (sitagliptin) & $\begin{array}{l}\mathrm{CrCl} 30-49: 50 \mathrm{mg} \text { daily } \\
\mathrm{CrCl}<30: 25 \mathrm{mg} \text { daily } \\
\text { HD/CAPD: no } \\
\text { supplement }\end{array}$ \\
\hline
\end{tabular}

${ }^{*}$ Dosing recommendations according to Epocrates Essentials (Epocrates Inc., San Mateo, CA).

${ }^{\dagger}$ Calculated by Cockroft-Gault equation.

$\mathrm{CrCl}$, creatinine clearance $(\mathrm{mL} / \mathrm{min})$; $\mathrm{HD}$, hemodialysis; CAPD, continuous ambulatory peritoneal dialysis.

tion recommends a target glycated hemoglobin level of $<7.0$ for all diabetics, regardless of whether kidney disease is present. ${ }^{8}$ Treatment of diabetes in patients with kidney disease can be complicated. Table 3 shows diabetic medications that need dosage adjustments by creatinine clearance. Metformin, which is the cornerstone oral medication for diabetic glycemic control, is contraindicated with creatinine $>1.5$ in men and 1.4 in women because of the concern about lactic acidosis.

\section{Tobacco Abuse}

Cigarette smoking is associated with a more rapid decline in kidney function regardless of the underlying cause of the chronic kidney disease. ${ }^{3}$ Smoking cessation should be discussed and encouraged in all 
Table 4. Screening Intervals for Complications of Chronic Kidney Disease by Stage

\begin{tabular}{lcrcr}
\hline Stage & $\begin{array}{c}\text { Complete } \\
\text { Blood } \\
\text { Count }\end{array}$ & $\begin{array}{c}\text { Intact } \\
\text { PTH }\end{array}$ & $\begin{array}{c}\text { Phosphorus/ } \\
\text { Calcium }\end{array}$ & $\begin{array}{r}\text { Total } \\
\text { CO }^{2}\end{array}$ \\
\hline 3 (GFR 30-59) & 12 & 12 & 12 & 12 \\
4 (GFR 15-29) & 12 & 3 & 3 & 3 \\
5 (GFR <15) & 12 & 3 & 1 & 3 \\
Dialysis & 12 & 3 & 1 & 1 \\
\hline
\end{tabular}

Values are presented as monthly intervals.

PTH, parathyroid hormone; GFR, glomerular filtration rate.

smokers with chronic kidney disease, particularly given that cardiovascular disease is the primary cause of mortality among this patient population. Medications used to facilitate smoking cessation, such as Zyban (GlaxoSmithKline, Inc., Research Triangle Park, NC) and Chantix (Pfizer, New York, NY), require dose adjustments in patients with renal disease; however, these can still be useful adjuncts in assistance with successful smoking cessation.

\section{Complications of Chronic Kidney Disease}

The prevalence of the complications of chronic kidney disease increases with each stage of the disease. According to the National Health and Nutrition and Examination Survey III database, ${ }^{15}$ the prevalence of complications for a patient with stage 1 chronic kidney disease is 0.28 , and it rises to an average of 1.71 in stage 4 . Therefore, it is recommended that screening for anemia, bone metabolism abnormalities, and metabolic acidosis be performed once the GFR is $<60 \mathrm{~mL} / \mathrm{min} / 1.73 \mathrm{~m}^{2}$ (stage 3$){ }^{3}$ The interval for each screening test varies by the stage of chronic kidney disease. Table 4 provides an overview of the screening recommendations from the National Kidney Foundation. If a complication is identified, the interval shortens to follow the success of treatment interventions.

\section{Anemia of Chronic Kidney Disease}

The National Kidney Foundation ${ }^{3}$ recommends that patients have at least an annual complete blood count as a screening for anemia. This recommendation leaves room for more frequent assessments in patients who are at higher risk or have had a decline in their hemoglobin but have remained within the normal range. ${ }^{3}$ The presence of anemia in a patient with chronic kidney disease requires further evaluation to elucidate the underlying cause of the anemia. Erythropoietin is produced by the kidney and stimulates production of reticulocytes in the bone marrow. As kidney function declines, the production of erythropoietin declines and anemia results. In addition, chronic kidney disease is a pro-inflammatory condition that can result in anemia of chronic disease. Furthermore, nutritional deficiencies may be present and the assessment of iron, folate, and vitamin $B_{12}$ levels should be made when clinically indicated. The minimal work-up suggested for a patient with anemia includes the complete blood count indices, reticulocyte count, serum ferritin, and transferrin saturation (TSAT). The reticulocyte count can be used to calculate the reticulocyte index, which provides essential information regarding the capability of the bone marrow to compensate for the anemia. The serum ferritin is a measurement of the total body iron stores and is considered low when $<25 \mathrm{ng} / \mathrm{mL}$ in men and $<12 \mathrm{ng} / \mathrm{mL}$ in women. The TSAT is a measurement of the adequacy of iron for erythropoiesis and is considered low when $<16 \%$. Classically, the anemia associated with chronic kidney disease is a normocytic, normochromic anemia with a normal ferritin and TSAT.

The target hemoglobin level for patients with anemia from chronic kidney disease is between 11.0 and $12.0 \mathrm{~g} / \mathrm{L}$. It is recommended by the National Kidney Foundation that hemoglobin should not be above $13.0 \mathrm{~g} / \mathrm{L}$ because this has been shown to be associated with increased incidence of cardiovascular mortality. ${ }^{3}$ There are several erythropoietin analogs that can be given either subcutaneously or intravascularly for the treatment of this anemia. Patients being treated with these agents require monthly assessments of hemoglobin levels because dose adjustments are frequently needed to maintain the hemoglobin at the target of 11.0 to $12.0 \mathrm{~g} / \mathrm{L}$. Work-up and treatment of any associated irondeficiency anemia should be done in conjunction with this treatment because the effectiveness of the erythropoietin is dependent on iron as a building block for hemoglobin synthesis.

\section{Bone Metabolism Abnormalities}

The National Kidney Foundation ${ }^{3}$ recommends that patients be screened for bone metabolism abnormalities with assessment of intact parathyroid hormone (PTH), phosphorus, and calcium at regular intervals, as directed by the stage of their 
Table 5. Target Phosphorus and Intact Parathyroid Hormone by Stage of Chronic Kidney Disease

\begin{tabular}{lcr}
\hline Stage & $\begin{array}{c}\text { Target Phosphorus } \\
(\mathrm{mg} / \mathrm{dL})\end{array}$ & $\begin{array}{r}\text { Target Intact } \\
\text { PTH }(\mathrm{pg} / \mathrm{mL})\end{array}$ \\
\hline 3 (GFR 30-59) & $2.7-4.6$ & $35-70$ \\
4 (GFR 15-29) & $2.7-4.6$ & $70-110$ \\
5 (GFR <15) & $3.5-5.5$ & $150-300$ \\
\hline
\end{tabular}

PTH, parathyroid hormone; GFR, glomerular filtration rate.

kidney disease (Table 4). These measurements should be done more frequently if the patient has an abnormality for which they are receiving treatment. The target values for phosphorus and intact PTH vary by stage of kidney disease whereas the target for calcium is the normal laboratory range (Table 5). Assessment of bone density with a dual energy radiograph absorptiometry scan for patients with chronic kidney disease is only recommended by the National Kidney Foundation for those patients with fractures or with known risk factors for osteoporosis. The US Preventative Services Task Force $^{16}$ agrees with this recommendation and does not list chronic kidney disease as a specific indication for osteoporosis screening.

Bone metabolism abnormalities are initially caused by elevations in phosphorus; renal excretion is impaired by the declining GFR. To compensate for the hyperphosphatemia, the parathyroid gland increases release of PTH. PTH has several effects on the kidney. It increases the absorption of calcium in the ascending limb of Henle, increases renal excretion of phosphorous by blocking reabsorption in the proximal tubule, and it activates 1-hydroxylase, which converts vitamin D to its active form: 1,25 hydroxyl vitamin D. PTH also acts on bone in conjunction with vitamin $\mathrm{D}$ by increasing the release of calcium and initiating the activation and proliferation of osteoclasts. Vitamin D has additional effects of increasing gut absorption of calcium and phosphorus as well as having a mild inhibitory effect on the release of PTH from the parathyroid gland. Secondary hyperparathyroidism is the compensatory increase in parathyroid production of PTH in response to the hypocalcemia and hyperphosphatemia caused by the declining renal function. Vitamin D deficiency results when the elevated PTH is unable to sufficiently stimulate the hydroxylation of $25-\mathrm{OH}$ vitamin $\mathrm{D}$ to its active form, resulting in a deficiency. Therefore, an ele- vated PTH level can signify secondary hyperparathyroidism, vitamin D deficiency, or both.

When serum phosphorus levels are elevated above the recommended range as determined by the patient's kidney disease stage, then treatment must be initiated. The first component of treatment is to restrict dietary phosphorus to 800 to $1000 \mathrm{mg}$ /day and to monitor blood work monthly to ensure the treatment is successful. ${ }^{3}$ If dietary adjustments alone are not sufficient then treatment with phosphate binders should be initiated. There are 2 broad categories of phosphate binders: calcium-based and noncalcium-, nonaluminum-, nonmagnesium-based phosphate binders. Both are effective in the treatment of secondary hyperparathyroidism. If calcium-based phosphate binders are used then, to avoid hypercalcemia, the total daily dose of elemental calcium in the medication should not exceed $1500 \mathrm{mg}$. $^{3}$

When serum intact PTH levels are elevated, either secondary hyperparathyroidism or vitamin D deficiency may be contributing. Therefore, before the initiation of treatment with dietary phosphate restriction and possibly phosphate binders, the serum level of 25-hydroxy vitamin D should be measured. If it is normal $(>30 \mathrm{ng} / \mathrm{mL})$ the screening should be repeated annually; however, if it is $<30$ $\mathrm{ng} / \mathrm{mL}$, supplementation with vitamin $\mathrm{D}_{2}$ (erocalciferol) should be initiated. ${ }^{3}$ Once the GFR declines into the level of stage 4 chronic kidney disease, there is significant decreased conversion of 25-hydroxy vitamin $\mathrm{D}$ to the active form of 1,25 hydroxy vitamin $\mathrm{D}$. Therefore, patients with stages 4 and 5 chronic kidney disease require replacement with 1,25-hydroxy vitamin $\mathrm{D}$ (calcitriol) or an active analog (paricalcitriol or doxecalciferol). ${ }^{17}$ Treatment of vitamin D deficiency can result in an elevation of calcium and/or phosphate, and these values must be monitored during treatment. If hypercalcemia $>10.2 \mathrm{mg} / \mathrm{dL}$ develops then treatment should be discontinued. Hyperphosphatemia can be treated with phosphate binders; however, if this does not successfully maintain target phosphate levels, vitamin D therapy may need to be discontinued.

The diagnosis of tertiary hyperparathyroidism should be considered in patients with known, longstanding secondary hyperparathyroidism who have become resistant to treatment. Tertiary hyperparathyroidism involves hypertrophy of the parathyroid glands that has developed into an autonomous ad- 
enoma. Treatment of tertiary hyperparathyroidism may require surgical parathyroidectomy.

\section{Metabolic Acidosis}

The National Kidney Foundation ${ }^{3}$ recommends that patients with chronic kidney disease be screened for metabolic acidosis by an assessment of total $\mathrm{CO}_{2}$ at varying frequency intervals per their stage of kidney disease (Table 4). The target total $\mathrm{CO}_{2}$ is $22 \mathrm{mmol} / \mathrm{L}$. When the level falls below 22 $\mathrm{mmol} / \mathrm{L}$, treatment with supplemental alkali salts should be considered.

\section{Cardiovascular Risk}

Family physicians must be aware that chronic kidney disease has been recognized as an independent risk factor for cardiovascular disease. Therefore, this patient population must be aggressively managed to prevent cardiovascular events. The modifiable risk factor of hyperlipidemia is of particular importance. Patients with chronic kidney disease frequently have multiple lipid abnormalities; however, low-density lipoproteins should be the primary target, with attention paid to the non-highdensity lipoprotein levels if the triglycerides are elevated to $>200$. The National Kidney Foundation and the National Cholesterol Education Program Adult Treatment Panel (ATP III) differ on their recommendations for chronic kidney disease patients. The National Kidney Foundation ${ }^{3}$ considers chronic kidney disease to be a coronary heart disease equivalent, placing all chronic kidney disease patients in the high-risk group as defined in the ATP III guidelines. However, the ATP III ${ }^{18}$ does not include chronic kidney disease in its calculation of hyperlipidemia goals. Given this information, it is prudent to have an minimum density lipoprotein goal of $>100 \mathrm{mg} / \mathrm{dL}$ for all patients with chronic kidney disease and an optional goal of $<70 \mathrm{mg} / \mathrm{dL}$, depending on the patient's additional risk factors or known cardiovascular disease status.

\section{Health Maintenance}

Family physicians play a key role in ensuring that patients with chronic kidney disease receive appropriate counseling and health maintenance interventions; this is a pivotal component to successful management of chronic kidney disease. Immunizations that are recommended for all chronic kidney disease patients are influenza and pneumovax vaccina- tions. ${ }^{19,20}$ Once a patient develops stage 4 chronic kidney disease, they should also receive the hepatitis B series because hemodialysis patients are at an increased risk of exposure to hepatitis $\mathrm{B}$, despite precautions. ${ }^{21}$ Additional health maintenance measures should be implemented according to the patient's other disease-specific recommendations and age.

Patients who smoke should be strongly encouraged to attempt to quit smoking because smoking is associated with increased cardiovascular disease and has been shown to accelerate the progression of chronic kidney disease. ${ }^{3}$ In addition, patients should be encouraged to follow a healthy lifestyle with exercise as a component to combat their increased risk of cardiovascular disease.

\section{Patient Education}

Patients need to be educated about their disease process so that their expectations are appropriate and they are active participants in their care. $\mathrm{Pa}$ tients need to be aware that over-the-counter medications (such as NSAIDs) are harmful to their kidney functioning and must be avoided. In addition, patients should be encouraged to bring all of their over-the-counter medications to their appointments for review because numerous herbal and weight-loss medications can also be nephrotoxic.

Patients with stage 4 chronic kidney disease need to be educated about the options of transplantation, hemodialysis, and peritoneal dialysis. These discussions should not be delayed until the point of impending dialysis and are best accomplished in conjunction with a nephrologist. It is known that morbidity and mortality are significantly improved when patients do not have to use temporary access for dialysis. ${ }^{22,23} \mathrm{~A}$ fistula should be placed approximately 6 months before anticipated need for dialysis to allow for maturation as well as any revisions that may be necessary. A graft, however, can be placed approximately 3 to 6 weeks before predicted time of need for dialysis. A peritoneal catheter should be placed 2 weeks before its anticipated time of need. Patients with stage 4 chronic kidney disease should also be counseled about not allowing blood draws or intravenous access, particularly subclavian lines or peripherally inserted central catheter lines in their nondominant upper extremity or other sites as dictated by imaging studies because 
the complications of these procedures may preclude the use of the involved extremity for future dialysis access.

The family physician needs to be ready to educate chronic kidney disease patients about these topics and provide preventive care and ongoing care to this patient population. Although referral to a nephrologist is always an option, family physicians should feel comfortable with making the initial diagnosis of chronic kidney disease and with providing appropriate initial and ongoing care to these patients.

\section{References}

1. Coresh J, Selvin E, Stevens LA, et al. Prevalence of chronic kidney disease in the United States. JAMA 2007;298:2038-47.

2. Israni RK, Shea JA, Joffe MM, Feldman HI. Physician characteristics and knowledge of CKD management. Am J Kidney Dis 2009;54:238-47.

3. National Kidney Foundation. K/DOQI clinical practice guidelines for chronic kidney disease: evaluation, classification, and stratification. Am J Kidney Dis 2002;39(2 Supple 1):S1-266.

4. National Kidney Foundation. Modification of Diet in Renal Disease study calculator. Available at www. kidney.org/professionals/tools. Accessed 9 September 2009.

5. Agency for Healthcare Research and Quality. US Preventive Services Task Force (USPSTF). Available at http://www.ahrq.gov/clinic/uspstfix.htm. Accessed 9 September 2009.

6. Chobanian AV, Bakris GL, Black HR, et al. Seventh report of the Joint National Committee on Prevention, Detection, Evaluation and Treatment of High Blood Pressure. Hypertension 2003;42:1206-52.

7. Mancia G, De Backer G, Dominiczak A, et al. 2007 Guidelines for management of arterial hypertension: The Task Force for the Management of Arterial Hypertension of the European Society of Hypertension (ESH) and of the European Society of Cardiology (ESC). Eur Heart J 2007;28:1462-536.

8. American Diabetic Association: standards of medical care in diabetes-2008. Diabetes Care 2008; 31(Suppl 1):S12-54.

9. Estacio RO, McFarling E, Biggerstaff S, Jeffers BW, Johnson D, Schrier RW. Overt albuminuria predicts diabetic retinopathy in Hispanics with NIDDM. Am J Kidney Dis 1998;31:947-53.

10. Hartman RP, Kawashima A. Radiographic evaluation of suspected renovascular hypertension. Am Fam Physician 2009;80:273-9.

11. Hunsicker LG, Adler S, Caggiula A, et al. Predictors in the progression of renal disease in the modification of diet in renal disease study. Kidney Int 1997; 51:1908-19.
12. Hebert CJ. Preventing kidney failure: primary care physicians must intervene earlier. Cleve Clin J Med 2003;70:337-44.

13. Mogensen CE, Neldam S, Tikkanen I, et al. Randomized controlled trial of dual blockade of reninangiotensin system in patients with hypertension, microalbuminuria, and non-insulin dependent diabetes: the candesartan and lisinopril microalbuminuria (CALM) study. BMJ 2000;321:1440-4.

14. Segura J, Ruilope LM. Should diuretics always be included as initial antihypertensive management in early-stage CKD? Curr Opin Nephrol Hypertens 2009; 18:392-6.

15. National Center for Health Statistics, Centers for Disease Control and Prevention. National Health and Nutrition Examination Survey. Available at http://www.cdc.gov/nchs/nhanes.htm. Accessed 9 September 2009.

16. Agency for Healthcare Research and Quality. Screening for osteoporosis in postmenopausal women. September 2002. Available at http://www. ahrq.gov/clinic/uspstf/uspsoste.htm. Accessed 9 September 2009.

17. Holick, MF. Vitamin D deficiency. N Engl J Med 2007;357:266-81.

18. National Cholesterol Education Program (NCEP) Expert Panel on Detection, Evaluation, and Treatment of High Blood Cholesterol in Adults (Adult Treatment Panel III). Third Report of the National Cholesterol Education Program (NCEP) Expert Panel on Detection, Evaluation, and Treatment of High Blood Cholesterol in Adults (Adult Treatment Panel III) final report. Circulation 2002;106:3143421.

19. Prevention of pneumococcal disease: recommendations of the Advisory Committee on Immunization Practices (AICP). MMWR Recomm Rep 1997; 46(RR-8):1-24.

20. Fiore AE, Shay DK, Broder K, et al. Prevention and control of influenza: recommendations by the Advisory Committee on Immunization Practices (AICP), 2008. MMWR Recomm Rep 2008; 57(RR-7):1-60.

21. Mast EE, Weinbaum CM, Fiore AE, et al. A comprehensive immunization strategy to eliminate transmission of hepatitis B virus infection in the United States: recommendations of the Advisory Committee on Immunization Practices (AICP) Part II: immunization of adults. MMWR Recomm Rep 2006; 55(RR-16):1-33, quiz CE1-4.

22. Chesser AM, Baker LM. Temporary vascular access for first dialysis is common, undesirable, and usually avoidable. Clin Nephrol 1999;51:228-32.

23. Lorenzo V, Martn M, Rufino M, Hernandez D, Torres A, Ayus JC. Predialysis nephrologic care and a functioning arteriovenous fistula at entry are associated with better survival in incident hemodialysis patients: an observational cohort study. Am J Kidney Dis 2004;43:999-1007. 